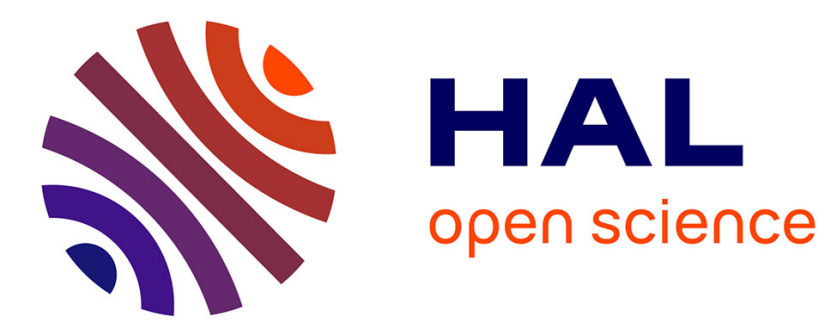

\title{
Le droit japonais comme traduction
}

Isabelle Giraudou

\section{To cite this version:}

Isabelle Giraudou. Le droit japonais comme traduction. Ebisu - Études Japonaises , 2011, 46 (1), pp.111-144. 10.3406/ebisu.2011.1706 . hal-03178755

\section{HAL Id: hal-03178755 \\ https://hal.science/hal-03178755}

Submitted on 24 Mar 2021

HAL is a multi-disciplinary open access archive for the deposit and dissemination of scientific research documents, whether they are published or not. The documents may come from teaching and research institutions in France or abroad, or from public or private research centers.
L'archive ouverte pluridisciplinaire HAL, est destinée au dépôt et à la diffusion de documents scientifiques de niveau recherche, publiés ou non, émanant des établissements d'enseignement et de recherche français ou étrangers, des laboratoires publics ou privés. 


\section{Le droit japonais comme traduction}

Isabelle Giraudou

\section{Citer ce document / Cite this document :}

Giraudou Isabelle. Le droit japonais comme traduction. In: Ebisu, n46, 2011. pp. 111-144;

doi : 10.3406/ebisu.2011.1706

http://www.persee.fr/doc/ebisu_1340-3656_2011_num_46_1_1706

Document généré le 30/07/2017 


\title{
Résumé
}

Cette étude propose une approche du droit japonais comme traduction. Elle envisage le paradigme traductif dans sa double acception, à la fois technique (le

droit traduit) et métaphorique (le droit comme traduction), et s'attache à préciser en quoi les mécanismes de (re) création juridique sont au Japon intimement liés à un ensemble de processus traductifs. Placer ainsi la traduction au coeur du droit japonais conduit à remettre en cause certaines analyses classiques (dont celles fondées sur les "transplants juridiques») et à réexaminer le caractère poreux d'un droit souvent qualifié trop lapidairement d' «hybride». De manière plus décisive encore, et en lien avec la mondialisation du droit, le paradigme de la traduction ouvre la réflexion juridique comparée à la notion d' "espace normatif».

\begin{abstract}
This article approaches Japanese law as translation. By examining legal translation in its technical as well as metaphorical aspects and Japanese law both as a translated law and as translation, it shows that legal transformation in Japan is a creative process closely linked to translation. By locating translation at the very heart of Japanese law we call into question the classical approaches dealing with the so-called •transfrontier mobility of laws" (in particular the wellknown theory of "legal transplants"). At the same time, this helps to explore further the supposedly "hybrid" character of Japanese law. Perhaps in an even more decisive way, and in relation with legal globalization, the translation approach may contribute to opening the comparative analysis to the notion of "normative space".
\end{abstract}




\title{
Le droit japonais comme traduction
}

\author{
Isabelle GIRAUDOU
}

Institut français de recherche sur le Japon (MFJ)

\section{Ouverture}

Rien, à première vue, ne prédestine un juriste frais émoulu (... de Paris II) à buter sur les mots. On l'a formé, sinon exclusivement au droit français, du moins à une certaine approche (française) du droit. On lui a aussi appris, avec plus ou moins de bonheur, à maitriser le lexique, la terminologie... le style qui vont avec - et à s'y tenir. Certains s'y tiennent - remarquablement. Davantage attirés par les chemins de traverse, d'autres tentent un écart - là encore avec plus ou moins de bonheur. Or, il arrive que ces chemins déportent de l'autre côté de la mappemonde - au Japon, par exemple. Et le " déserteur » de se retrouver alors « sans gouvernail sur une mer océane » (Sugita 1982 : 38) : entre au moins deux pays, deux histoires, deux pensées, entre "plus d'un droit "; et ayant à risquer, d'abord, cette pérégrination dé-routante d'une langue à une autre.

Dix ans plus tard : le roi est enfin nu. ... Et toujours sur le fil d'une langue d'adoption.

Tant « il faudrait inventer des mots nouveaux» - m'écris-tu.

\ Isabelle Giraudou est chercheure à l'Institut français de recherche sur le Japon (MFJ, UMIFRE 19 CNRS-MAEE), chercheure associée de l'Institut d'Asie orientale (UMR 5062 CNRS) et membre du réseau « Mondialisation du droit - Global Legal Studies Network» (FMSH Paris). 


\section{Problématique}

Le droit entretient un rapport consubstantiel à la langue, au langage, aux pratiques langagières, et aux formes linguistiques; la langue juridique est sa "première enveloppe" (Capitant 1930: 7). La question de la traduction en droit, qui connaît un regain d'intérêt parmi les juristes (Cornu \& Moreau 2011) ${ }^{1}$, conforte ce constat d'évidence tout en le compliquant. Il est certes acquis que la traduction d'une langue à une autre accompagne les transformations internes de nombre de systèmes juridiques tout autant que le développement de leurs interactions - a fortiori sous la poussée de certains facteurs, dont la "globalisation ». Mais l'approche du "droit comme traduction " va plus loin que l'analyse somme toute classique et confinée de la " traduction du droit ", et vient enrichir l'ensemble des théories concernant ce que l'on appelle - par raccourci intellectuel, et faute encore de mieux - la " circulation du droit " : elle illustre en effet au plus près ce changement de paradigme ou cette rupture épistémologique qui implique pour la pensée juridique de passer d'un modèle inspiré par les sciences de la nature ("transplants juridiques ", "greffes ", ...) à un modèle proche des sciences du langage ${ }^{2}$. De nature différente, la réflexion développée à partir de l'hypothèse du droit comme traduction revêt ainsi une tout autre portée. Or, si les études portant sur la «traduction du droit » abondent, celles opérant le saut épistémologique d'une approche du droit comme traduction se comptent sur les doigts d'une seule main. Si peu de systèmes juridiques se prêteraient-ils donc à une telle lecture?

Les premiers théoriciens du droit à s'être risqués sur ce terrain... sont japonais. La question du droit comme traduction apparaît au Japon indissociable d'une réflexion plus vaste sur ce que l'on appelle la « réception» du

1. Ainsi du colloque organisé les 15 et 16 octobre 2009 par le Centre d'études sur la coopération juridique internationale (CECOJI - UMR 6224 CNRS) et Juriscope (Accès aux droits étrangers et promotion du droit français et des droits francophones - UMS 2268 CNRS) à la Faculté de droit et des sciences sociales de l'université de Poitiers, dont les actes ont été publiés sous le titre Traduction du droit et droit de la traduction (Cornu \& Moreau 2011).

2. Pour un aperçu du registre métaphorique utilisé par les comparatistes pour décrire la « circulation du droit », voir : Örücü 2002 : 7 et s. ; Nelken 2002 : 19-34 ; Giraudou 2011b. 
droit. Car c'est sur ce point que le bât blesse encore. La réflexion développée par le comparatiste francophone Noda Yoshiyuki 野田良之 à propos de la traduction fait ainsi écho à son interrogation sur la réception du droit au Japon (keiju 継受), objet d'une "croyance commune " : " on y croit ", mais y a-t-il réellement eu "réception ", est-il pertinent de parler en ces termes? Car «si fidèlement et habilement que [le Japon] ait réussi à imiter les systèmes juridiques [de la France et de l'Allemagne], son propre milieu culturel ne laisse pas de donner au système qu'il a reçu - malgré l'apparente analogie - un caractère original " (Noda 1966 : 65). La question restant de savoir comment penser cette " originalité » du droit japonais et la singularité de son expérience historique en matière de réception.

Le système juridique japonais est diversement qualifié. Mais les analyses le rattachant aux systèmes de droit civil (en raison de son caractère codifié) ou le rapprochant des systèmes de Common Law (en raison de l'influence du droit américain) éludent la plupart du temps la question de savoir comment et dans quelle mesure s'articulent en son sein différentes traditions juridiques. D'autant que les travaux introduisant au droit japonais s'en tiennent en général à une vision chronologique, et appréhendent la formation du droit japonais moderne principalement en référence à une circulation plane ou nationelle du droit (en provenance soit de la France, soit de l'Allemagne, ... $)^{3}$. Quant aux auteurs qui en vantent d'emblée le caractère " hybride ", ils le font plutôt par facilité de langage, sans qu'une réflexion approfondie vienne étayer une qualification séduisante sans doute, mais creuse. De sorte que l'hybridation attendue (et a posteriori tant vantée) entre différentes traditions juridiques n'aurait pas encore été réellement pensée en doctrine...

Le comparatiste francophone Kitamura Ichirō 北村一郎, à qui la traduction au Japon apparaît « moins comme un phénomène technique localisé à l'intérieur de la pratique juridique ou judiciaire que comme relevant du système juridique tout entier ", tente de restituer au traducteur son troisième visage : pas seulement celui de l'emprunt et de la traîtrise épistémologique,

3. Voir Kasai 2009 ; Matsumoto 2010 : 110-128 ; ainsi que le résumé de leurs interventions respectives lors du colloque international Global Law and Global Legal TheoryAcademic Knowledge in Question (MFJ, Tokyo, 3-4 juin 2001), pp. 22-30 ; compte rendu en ligne : http ://www.mf.gr.jp/web/sympo_20110603/pdf/CR-IW_3-4_June_2011. pdf. 
mais aussi celui de la création - car il n'est pas exclu que le traducteur devienne trovatore (Kitamura 1987 : 753). En formulant l'hypothèse d'un "droit créole », le philosophe du droit Hasegawa Kō 長谷川晃 va plus loin encore dans la perception de la traduction (et de la supposée " réception ») comme acte de création normative 4 . En renouvelant cette science des mots (Wortwissenschaft, Pound 1954) qu'est le droit comparé, l'hypothèse formulée par Hasegawa - pertinente malgré un cadre d'analyse assigné (principalement historique) et certains partis pris méthodologiques - permettrait-elle d'aller au-delà de la seule remise en cause des taxinomies classiques du droit comparé ? D'un droit traduit à un droit comme traduction : le saut tenté est d'ordre épistémologique. Qui donne de mieux saisir en quoi la traduction - marque d'hospitalité langagière - dessine les contours d'un nouvel espace de sens, et (pierre d'angle ?) construit une langue juridique " commune ", susceptible de « réconcilier » différentes traditions, pratiques, et pensées du droit. Placer ainsi la traduction au cœur même du droit japonais conduit non seulement à remettre en cause certaines analyses classiques et à réexaminer son caractère " hybride " mais, et de manière plus décisive encore, permet d'ouvrir la réflexion juridique comparée à la notion d'" espace normatif ${ }^{6}$ ».

4. Notons que, depuis déjà plusieurs années, une série de publications et de manifestations variées (universitaires, sociologiques, littéraires, philosophiques et médiatiques) a pu faire porter au mot "créole " les questionnements les plus actuels de la société japonaise. Pour l'écrivain Michaël Ferrier, le Japon - tout en restant un cas excentré, et à bien des égards déplacé dans le réseau labile des études créoles - n’en apparaît pas moins comme le lieu où s'élaborent de nouveaux enjeux de pensée autour de la figure du Créole. Instrument intellectuel autant qu'arme politique, les théories de la créolisation reflètent d'une part un positionnement stratégique dans le champ académique et, d'autre part, une réflexion émergente sur la place du Japon dans la sphère internationale. Et c'est principalement pour penser les mutations du Japon moderne, son rapport au monde "globalisé ", et ses tentatives d'articuler l'hétérogène, qu'elles sont formulées (Ferrier 2009 : 75-202).

5. Un rapprochement - aussi audacieux qu'éclairant - avec la réflexion de Clarisse Herrenschmidt sur l'écriture m'a ainsi été suggéré par Gilles Lhuilier.

6. Cette étude décrit les principaux points d'une réflexion en cours ; étape intermédiaire dans mon travail rédactionnel, elle s'inscrit dans un travail collectif de réflexion conduit autour de la notion d' " espace normatif " telle qu'initiée par Gilles Lhuilier, et résume certains développements de l'ouvrage en cours de co-rédaction, Le droit japonais des sociétés: un droit global? Réflexion à propos d'un changement de paradigmes (titre 


\section{La traduction du droit au Japon}

\section{Une culture de la traduction?}

\section{a. La traduction au Japon : un concept flou}

Les références à l'importance de la traduction au Japon abondent. Force est pourtant de constater que la " théorie de la traduction " n'y est pas considérée comme une discipline scientifique ou académique à part entière : la traduction resterait un " concept flou ". De même, dans le domaine juridique, l'appétence pantagruélique pour le travail traductif - indéniable - est peu interrogée en tant que telle. Comme si la question de la traduction en droit était encore nimbée d'un "silence parfait et optimiste " (Kitamura 1987 : 751). Les rares juristes japonais à s’être penchés sur la traduction de certains mots (termes, concepts) ne sont pas les spécialistes d'une branche spécifique du droit positif japonais, mais plutôt ces explorateurs opérant à la périphérie des programmes universitaires que sont - au Japon aussi - les philosophes ${ }^{7}$, sociologues et historiens du droit (tous étant, le plus souvent, comparatistes).

Ceci étant, la réflexion de fond développée par l'historien et spécialiste de théorie politique Maruyama Masao 丸山真男 et l'écrivain et critique littéraire Katō Shūichi 加藤周一 est, pour les juristes aussi, susceptible de faire date. Leur dialogue, publié sous le titre Hon.yaku to Nihon no kindai 翻訳と

provisoire). Lors d'un colloque international (juin 2009, Maison franco-japonaise), Gilles Lhuilier (professeur de droit privé, université Bretagne-Sud) présentait la notion de décentrement et développait - s'agissant d'un objet spécifique : les " contrats globaux " - une réflexion sur les « nouveaux espaces normatifs transnationaux ", l'articulation possible de différents espaces de normativité, et l'émergence d'une langue internationale des contrats. Intitulé " Global Law and Global Legal Theory - Academic Knowledge in Question ", le colloque international organisé les 3 et 4 juin 2011 (Tokyo) par la MFJ - avec le soutien du Clarke Program in East Asian Law and Culture, Cornell University, et la participation du Global Legal Studies Network - a permis de poursuivre la réflexion et d'interroger sur ce fondement notamment les transformations du comparatisme en droit.

7. Voir Uehara Mayuko : ouvrages référencés dans la bibliogaphie. Significativement, celui qui a poussé le plus loin la réflexion sur cette question dans le domaine juridique est un philosophe du droit, comme nous verrons. 
日本の近代 (Maruyama \& Katō 1998), et faisant suite à Hon.yaku no shisō 翻訳の思想 (Maruyama \& Katō 1991), présente pour la première fois une analyse macroscopique de la traduction. Les auteurs y voient un élément décisif de la modernité japonaise, et soulignent que seule une approche pluridisciplinaire - mêlant histoire, sciences politiques, relations internationales, littérature, études culturelles, théorie du langage, etc. - permet d'appréhender les implications de la traduction pour le Japon moderne. À cheval sur les frontières disciplinaires, le tableau qu'ils brossent les conduit à repenser l'" ouverture " historique du pays sous Meiji à partir de l'histoire de la traduction. Et à définir le Japon comme une " culture de la traduction".

\section{b. Des kanji comme outils de traduction}

La culture de la traduction n'a pas germé ce jour de 1853 où les kurofune 黒船 du commodore Matthew Perry franchirent la ligne d'horizon, « invitant " le Japon à mettre fin à 250 ans d'isolement artificiel, mais elle plonge ses racines dans l'histoire longue du pays. Le rapport de grande proximité des Japonais avec l'écriture chinoise et la complexité des arrangements auxquels ils ont procédé pour faire leurs les kanji, ainsi que les contacts répétés du Japon avec les langues occidentales à partir du XVI ${ }^{\mathrm{e}}$ siè$c^{8}{ }^{8}$, devaient en effet prédisposer au projet traductif qui sera celui de l'ère Meiji. Indissociable de techniques spécifiques de lecture et d'écriture, l'œuvre de traduction effectuée au XIX ${ }^{\mathrm{e}}$ siècle s'appuie sur un processus séculaire. Éclairante, l'analyse de ces techniques intéresse aussi une réflexion sur le droit comme traduction.

De l'écriture à la lecture - Des kanji comme outils de traduction ? Le sujet n'a que très rarement été abordé par la traductologie occidentale

8. La première rencontre du Japon avec une langue occidentale (portuguais) date du milieu du XVI ${ }^{\mathrm{e}}$ siècle. Au siècle suivant, le nombre d'interprètes en néerlandais (Oranda tsūji オランダ通事) s’accroît, certains développant une réflexion méthodologique sur la traduction : Motoki Yoshinaga 本木良永 (1735-1794) ou Shizuki Tadao 志筑忠雄 (1674-1728). En 1808, le shogunat encouragea l'étude de l'anglais. La multiplication des échanges avec la Russie favorisa l'apprentissage du russe et, conséquemment, du français. 
(Brotherston 1998 : 211). Or, formuler l'hypothèse d'une « culture de la traduction » à propos du Japon suppose d'examiner son lien avec l'histoire des signes - la question étant pour nous plus spécialement de savoir en quoi l'écriture japonaise (davantage qu'aucune autre peut-être) contribuerait à faire de la traduction en droit un acte de création normative. Quelques précisions s'imposent ici à titre préliminaire.

L'écriture, tout d'abord. L'écriture japonaise s'écrit au moyen d'une combinaison de caractères lui permettant " d'une manière ou d'une autre [de] se traduire" (Noda 1966 : 18) : l'écriture, qui rend la langue visible, est déjà traduction ; l'écriture japonaise l'est doublement dans la mesure où son histoire renvoie à un déploiement sémiologique spécifique, lié à l'expansion graphique de la Chine. Rappelons que, en l'absence de tout système d'écriture préexistant, les caractères idéographiques (kanji) ont été introduits au Japon - via la Corée - entre les $I^{e}{ }^{e}$ et $v^{e}$ siècles de notre ère. Ils furent d'abord utilisés pour les registres financiers et diplomatiques de la Cour, la tâche de scribe étant alors confiée à des descendants d'immigrés coréens et chinois. Quant à l'étude des classiques chinois, elle était surtout le fait d'une frange nobiliaire, davantage intéressée par la compréhension du contenu de ces textes que par la maîtrise des techniques du chinois comme langue source. Dans le Japon ancien, il ne s'agissait donc pas tant de produire un texte parallèle rédigé à l'aide d'une écriture différente (qui n'existait pas) - que de lire en japonais ces textes dans leur version originale. Qu'est-ce à dire?

Rappelons que l'on distingue au Japon au moins deux types de lectures des kanji : les lectures phonétiques, on-yomi 音読み, basées sur une prononciation la plus approchante de la prononciation chinoise historique des caractères $^{9}$; et les lectures proprement japonaises des kanji, ou kun-yomi 訓読み (lecture explicative), consistant principalement à assigner des mots autochtones japonais (et leur prononciation) à des idéogrammes ayant en Chine une signification analogue. Couramment pratiqué par l'élite japonaise depuis le IX jusqu’à la fin du XIX ${ }^{\mathrm{e}}$ siècle, le kanbun kundoku 漢文訓読,

9. Inspirée de la pratique bouddhique consistant à réciter les sūtra en chinois, et en usage principalement dans les universités, la lecture en chinois des idéogrammes n'était cependant pas courante et ne signifie pas que les Japonais de l'époque maîtrisaient cette langue ; voir Semizu 2006 : 290. 
ou " lecture japonaise de l'écriture chinoise », avait été initialement élaboré pour permettre la transmission orale des textes bouddhiques et leur diffusion auprès du clergé japonais (Ueda 2011 : 141-142). Technique de lecture explicative et interprétative, le yomikudashi 読み下し consiste : à inverser mentalement l'ordre des mots du texte source (en suivant ou non certaines indications scripturales, marqueurs liés à la langue cible, ou kunten 訓点) ; à rendre le texte conforme aux exigences grammaticales et syntaxiques de la langue japonaise (moyennant, notamment, l'ajout de particules); et à le prononcer en japonais. Visuellement, les textes portant l'empreinte de cette technique de lecture donnent «l'illusion » au lecteur d'accéder directement au texte chinois original : le kanbun kundokutai 漢文訓読体 permet ainsi de lire « en langue locale " les classiques chinois.

En particulier s'agissant de concepts abstraits et des termes associés, il arrive cependant que l'écart sémantique entre l'idéogramme chinois et son kun japonais se creuse considérablement - il ne s'agirait alors pas d'une " rencontre entre deux cultures différentes à un niveau compatible " (Mizuta 1968 : 2). Deux lectures différentes d'un texte - et donc deux textes différents ? Certes, concernant le rapport - plus ou moins élastique - de proximité (ou de force...) entre le caractère chinois, sa signification originale, sa prononciation et le sens assigné en japonais, les approches different (Nakata 1949: 43 ; Yoshino 1992 : 51). Toujours est-il que ce rapport reste, de manière caractéristique, indéfini, non systématique (Kamei et al. 1963 : 252). C'est sans doute pour cette raison que, très tôt appréciée par le Japon, la " capacité d'expression " des kanji poussera les traducteurs à utiliser ces "prétendus idéogrammes " pour tenter d'exprimer le sens de certains mots occidentaux $^{10}$ (Yanabu 2009 : 19). L'analyse des techniques de lecture des caractères chinois éclaire ainsi combien, paradoxalement, cette « culture de la traduction » a oscillé entre cécité à la traduction et refus de traduire.

10. Le Japon créa en outre quelque 1500 nouveaux caractères (kokuji 国字, ou « caractères nationaux "). Composés chacun de 48 signes, les syllabaires japonais katakana et hiragana (systèmes d'écriture et de lecture phonétiques) tels qu'utilisés aujourd'hui furent pour leur part élaborés au VIII ${ }^{\mathrm{e}}$ siècle ; ils permettent une retranscription en japonais sans le recours aux caractères chinois. 
La traduction rendue visible - Ou lire en japonais l'écrit chinois (kanbun kundoku). Aux yeux de ses contemporains qui croyaient lire les Analectes de Confucius et le Livre de Mencius en chinois, le penseur confucianiste Ogyū Sorai 荻生徂徠 (1666-1728) sera celui par qui le scandale arrive. En substance : « le lecteur japonais est traducteur; ce qu'il lit est la traduction ; l'original est en langue étrangère "; le chinois n'est pas le japonais - il est traduit en japonais ; du chinois (classique) au japonais, existe un rapport traductif. Sorai démasque ainsi la traduction, alors qu'elle s'abrite derrière le double voile d'un emprunt séculaire (à la terminologie comme à l'écriture chinoises) et de cette stratégie spécifique de lecture qu'est le yomikudashi (voir plus haut). Pour Maruyama, Ogyū Sorai est le premier érudit à avoir pensé cette convention de lecture comme acte de traduction ${ }^{11}$. Ce que déplore Sorai n'est pas tant la technique du yomikudashi que la cécité de ses contemporains à la traduction. Lui œuvre pour que l'acte de traduction ne soit pas un acte manqué : faire consciemment l'écart de la langue, via la traduction (par rapport au chinois, langue étrangère) devant ouvrir l'espace de la comparaison. Le "multilinguisme " en cours dans le Japon de la fin des Tokugawa a pu éveiller la conscience de Sorai à ce rapport traductif entre le chinois et le japonais. Maruyama souligne, du point de vue " méthodologique ", ce trait de génie qui consiste pour Sorai à rapprocher le chinois du hollandais (via les études hollandaises, ou Rangaku 蘭学) pour penser le rapport de traduction existant entre la langue japonaise et les langues étrangères, chinois inclus : le japonais devient alors une langue parmi d'autres; la traduction en japonais permettant de se rendre compréhensibles... y compris « le babil des barbares et les cent langues de la pie-grièche ${ }^{12}$ ».

En théorie, la question de savoir si la lecture kanbun kundoku constitue ou non une "traduction» reste discutée, les auteurs s'accordant à penser que le kanbun kundoku est à la fois plus et moins qu'une traduction au sens

11. Ogyū Sorai aborde pour la première fois la question de la traduction dans son ouvrage Yakubun sentei 訳文鉒蹄 (Guide de la traduction), publié en 1715. Ce " guide " - présenté sous la forme d'un dictionnaire listant les mots chinois devenus homonymes lors de leur passage en lecture japonaise, et expliquant leur signification originale - met en lumière l'écart de signification entre le mot ou l'idéogramme chinois et sa lecture japonaise (écart d'autant plus creusé qu'un même idéogramme peut faire en japonais l'objet de plusieurs lectures/prononciations).

12. Cité dans Maruyama \& Katō (1998 : 29-30). 
conventionnel. Pour certains juristes, cette technique de lecture constitue bien un acte de traduction (Noda 1975 : 693), tandis que pour d'autres il s'agit plutôt d'une technique spécifique d'assimilation permettant justement de n'avoir pas à traduire le chinois (Kitamura 1987 : 762). Nous lisons non l'original, mais le texte traduit. Ce rappel, qui ébranla si fortement les contemporains de Sorai, est aussi de nature à élargir la compréhension que nos contemporains (occidentaux) se font de la traduction. Continuant de hanter le comparatiste, cette rupture soraienne - ou dévoilement - constitue le point de départ de la réflexion juridique ici présentée.

Le retrait de la traduction - $\mathrm{Ou}$ " lire en chinois l'écrit chinois ». Tandis que le kanbun kundoku désigne la technique de lecture japonaise des textes chinois, le ondoku 音読 consiste à lire ces textes dans le respect de la syntaxe originale et selon une prononciation rendue aussi ressemblante que possible à la prononciation chinoise. Technique de lecture couramment utilisée dans la récitation des sütra bouddhiques, ce style curieusement s'avéra des plus malléables, permettant de former de nouveaux composés ou de nouvelles combinaisons de kanji pour " traduire " les mots étrangers, singulièrement les mots occidentaux. On retrouvait ce style non seulement dans les colonnes de journaux ou les traductions d'ouvrages étrangers, mais aussi dans les déclarations et les nouveaux textes juridiques de l'époque. Fût-ce au prix d'une certaine opacité pour les moins lettrés ${ }^{13} \ldots$

Ce que Yanabu Akira 柳父章 appelle « effet cassette» (kasetto köka カセット 効果), ou assomption de sens engendrée par un kanji, apporte ici un complément intéressant (Yanabu 1982, 2009). Selon le théoricien de la traduction, les mots étrangers ont toujours joui au Japon d'un prestige les faisant paraitre d'emblée tel un coffret dont on anticipe (comme nécessairement précieux) le contenu ; la fascination exercée par l'enveloppe des mots précède la compréhension de leur signification - voire s'y substitue. Yanabu voit dans le ondoku un refus de traduire, la renonciation même à toute

13. Les intellectuels, prompts à élaborer de nouvelles combinaisons de kanji, failliront à la tâche de diffuser les idées nouvelles auprès des couches populaires. Le caractère " impraticable " et opaque des (nouvelles) combinaisons - accessibles seulement à une élite plurilingue - jouera comme argument initial dans la campagne anti kanji développée sous Meiji. 
recherche d'équivalence (que suppose la quête d'une langue unique) - par extension caractéristique, selon lui, du rapport japonais aux textes étrangers. Cette réflexion sur la traduction conduite à partir de la lecture ondoku lui permet de mettre en lumière l'existence d'un " écart traductif ", ou rapport d'asymétrie entre la signification du mot d'origine et l'opacité de sa traduction en japonais : la médiation des kanji pour traduire en japonais les mots d'origine occidentale permet de former des néologismes qui ne renvoient pas nécessairement au sens du mot d'origine - voire le rendent opaque et à jamais inaccessible. Si son analyse intéresse la présente étude, c'est moins en raison de l'accent mis sur l'existence d'un " écart traductif " en tant que tel (Yanabu n'adopte d'ailleurs pas la perspective d'une langue universelle, sorte de lexique pré-babélien, ni n’appréhende les écarts traductifs comme autant d' " erreurs de traduction " par rapport à une langue correcte). Mais plutôt en ce que ce " refus de traduire " pondère l'idée de "traduction conceptuelle", tout en faisant voler en éclats l'approche d'un rapport traductif supposé linéaire (et descendant) entre une source active (toute signifiante) et une cible passive (nécessairement réfléchissante, fût-ce sur le mode inversé du malentendu).

Ces analyses gardent pour nous tout leur intérêt. En s'inscrivant résolument en faux contre les approches dichotomiques de la traduction, elles soulèvent directement la question d'un "interlangage " : loin de consister en un transfert latéral (et a fortiori a-éclectique) d'une langue source strictement étrangère à une langue cible étroitement autochtone, le processus de traduction renvoie au contraire à l'élaboration d'une "langue tertiaire " (ni radicalement extérieure, ni complètement domestique). Inaugurales, elles fournissent à la présente étude un éclairage précieux : soulevant la question de savoir en quoi la langue-écriture japonaise fait de la traduction en droit un acte de création normative, je tente ainsi d'approcher la traduction au Japon plus largement qu'au seul sens technique du terme, pour en sonder la signification et la portée dans le domaine juridique. 


\section{Une culture juridique de la traduction?}

La traduction en japonais marque tous les âges de la vie du droit japonais ${ }^{14}$. La modernisation de ce droit et ses transformations actuelles pourraient même être présentées comme indissociables du traductionisme. Kitamura Ichirō évoque ainsi à propos du droit japonais sinon " un monde tout traduit ", du moins " une cité pavée d'une mosaïque plurilingue souterraine " (Kitamura 1987 : 750). Une première description du travail historique et contemporain de traduction éclaire ce "plurilinguisme occulte».

\section{a. Un droit traduit}

La traduction en langue japonaise de termes juridiques occidentaux effectuée par les intellectuels de l'époque ${ }^{15}$ a commencé dès la fin de la période d'Edo $^{16}$ : en 1841 , le shogunat ordonnait à certains spécialistes japonais de traduire des textes juridiques hollandais; et à la fin des années 1850, une traduction systématique de la terminologie juridique et institutionnelle européenne et de la terminologie du droit international était entreprise dans le cadre de l'Institut pour l'investigation des écrits étrangers (Bansho shirabesho 蕃書調所). "Faire l'expérience de l'Occident » consiste aussi à effectuer, dès la fin du bakufu 幕府, une sortie de l'Archipel pour aller au-devant des juristes européens et américains ${ }^{17}$; et à accueillir au

14. La place manquant ici, je n'évoquerai que brièvement la question de la traduction en japonais, et renvoie à mon étude préliminaire pour compléments et examen de la question d'une traduction du droit japonais en langue étrangère (Giraudou 2011a).

15. Les intellectuels missionnés - dont la plupart sont issus de familles de guerriers et deviendront membres du nouveau gouvernement - ont une double formation : en droit et en langue étrangère (le chinois et le hollandais, d'abord ; puis l'anglais, le français, et l'allemand). Grâce à un certain nombre d'ouvrages déjà traduits et publiés en Chine ou au Japon, ils ont pu se familiariser dès le début des années 1850 avec le vocabulaire juridique et politique occidental, notamment en procédant sur la base du hollandais ou du chinois à des associations ou connexions de sens.

16. La traduction des termes juridiques chinois est antérieure, le Japon ayant commencé de s'inspirer de la législation des codes (ritsuryō 律令) mise en place par la Chine au milieu du viI ${ }^{\text {e }}$ siècle ; voir Hérail (2008).

17. Voir, par exemple, le remarquable ouvrage de Takii Kazuhiro 滝井一博 (2003, [traduction anglaise] 2007). 
Japon même et sur invitation officielle des oyatoi gaikokujin 御雇外国人 (ou conseillers étrangers).

La traduction juridique au Japon constitue aussi une préoccupation contemporaine. Sur le plan doctrinal, et en particulier en ce qui concerne l'interprétation des textes, l'œuvre historique de réception implique a minima un " contrôle continu avec [l'] original " (Kitamura 1987 : 750). De même, les réformes législatives au Japon sont en général précédées d'une recherche comparative conduite au niveau bureaucratique ou, plus souvent encore, par les universitaires.

\section{b. Une comparaison-traduction}

$\mathrm{Au}$ Japon, la traduction imprègne très fortement le rapport au droit comme discipline : on y est rarement juriste sans être aussi " comparatiste "; voire rarement comparatiste sans être aussi «traducteur». Pourtant, la traduction - en japonais et $d u$ japonais - a parfois moins concouru à la transparence de ce droit, qu'elle n'en a cultivé la relative opacité. Rencontre de l'étranger dans sa propre langue, la traduction reste une épreuve. Et permet la comparaison autant qu'elle l'hypothèque.

... hors contexte - Selon Kitamura Ichirō, la traduction en japonais fait filtrage en ce que seul l'explicite est traduit. L'implicite (la dimension culturelle, socio-économique, philosophique, voire religieuse et métaphysique du droit) est extrait pour être laissé de côté, tel un résidu. La traduction procède par voie de décontextualisation: elle condense, ne retenant que la part la plus technique du droit. Avec pour double effet d'enclore la science juridique elle-même à l'intérieur d'un système " tout fait, suffisamment défini », la poussant sur la voie d'un " dogmatisme subtil » (Kitamura 1987 : 788) - et d'une opacité croissante pour les non-initiés. Le processus de décontextualisation sur lequel repose la traduction au Japon n'est pas sans risque; le prix à payer est double : renforcement de l'effet de mystification et accroissement prévisible de la désaffection des justiciables; déracinement intellectuel d'une pensée juridique ballotée entre "xénomanie " d'un côté, chauvinisme de l'autre. Cette réflexion ne condamne pas l'œuvre de traduction, mais dirige la critique vers la science juridique elle-même. Critique vis-à-vis d'une approche étroitement positiviste, Kitamura Ichirō appelle 
ainsi les juristes contemporains à être à la fois sociologues et comparatistes avertis, attentifs à la pluralité des « visions du monde ».

... muette - Une hypothèse voudrait que, en l'absence plus ou moins voulue de traduction en direction de l'étranger, la comparaison n'opère que dans un seul sens : les comparatistes japonais, pourtant si soucieux euxmêmes de traduire en japonais des pans entiers de droit et de doctrine juridique étrangères, s'ingénieraient à ne pas traduire leur propre droit. En ne se livrant qu'à reculons dans la langue de l'autre, le droit japonais redeviendrait étroitement archipélagique, ne circulant plus dès lors qu'en vase clos... Certains auteurs pointent ainsi la difficulté qu'ont les comparatistes japonais à organiser en sens inverse la circulation de leur propre réflexion comparée, voire stigmatisent un réflexe de fermeture. Le traductionisme à sens unique aurait-il paradoxalement rendu le droit japonais opaque? Selon Kasai Yasunori 葛西康徳, loin d'avoir œuvré à la transparence du système juridique, la traduction a au contraire eu pour effet d'enclore ses transformations dans le seul environnement linguistique japonais, de le verrouiller sur lui-même (Kasai 2009 ; 2010). Ironie : la traductio (" traversée ") a échoué à tracer dans l'espace discursif un lieu de contact. À l'abri de leur propre langue (relativement peu parlée par la communauté des juristes en dehors de l'Archipel), les comparatistes japonais procèdent indépendamment de toute interférence ou censure externes. La traduction maintient l'autre partie à distance et soustrait son résultat à toute (re)discussion possible. Troika juridique? Voyage en tout cas sans retour, avec pour conséquence de rester en retrait de l'échange traductif ${ }^{18}$; devenue unilatérale, la traduction reste un exercice hermétique à la comparaison, et pour un peu : stérile. Or, le plus souvent ignorants de la langue d'origine, les destinataires de la

18. Observons toutefois que cette attitude contraste avec celle de certains " pionniers ". 1889 : Kaneko Kentarō 金子堅太郎, assistant d’Itō Hirobumi 伊藤博文 (qui, la même année, démissionna de la présidence du Conseil privé Sūmitsu-in 枢密院 et reçut le titre honorifique de daijin 大臣 par ordre impérial), prend ainsi la route de l'Europe, cette fois pour soumettre à l'examen le projet de Constitution à la rédaction duquel il participa. Il emporte avec lui la version anglaise de la nouvelle Constitution du Japon et les Commentaires sur la Constitution, interprétation officielle participant d'un double mouvement : appropriation (par la doctrine japonaise) et explicitation (à destination des juristes occidentaux). 
traduction (les sujets de droit, ses utilisateurs) ne peuvent que faire aveuglément confiance au traducteur - d'aucuns mettant alors en garde contre les risques d'un "droit-ésotérique, réservé au pouvoir et à ses mandarins ", et provoquant davantage encore " la mystification" (Kitamura 1987 : 791). Ces observations ne ruinent pas la possibilité de tout échange traductif, mais encouragent à rompre avec la pratique d'une traduction-comparaison polarisée - effectuée au prisme d'une spécialisation dans un droit étranger (et dans la langue qui lui est associée), puis frileusement maintenue en repli (comme silencée au sein de la langue maternelle).

... monologique - Une autre hypothèse voudrait que, en dépit de la traduction, la comparaison n'opère pas. Présenter son propre droit dans une langue étrangère ne conduirait-il pas le comparatiste japonais à parler, en définitive, d'un autre droit ? Plusieurs auteurs se sont montrés sensibles à un tel écueil, mais Kitamura Ichirō fut l'un des premiers à formuler sans ambage cette réserve pour mieux y sensibiliser ses collègues français et japonais francophones; ainsi, lors des IV $\mathrm{V}^{\text {es }}$ Journées juridiques franco-japonaises, ce dernier faisait-il part de son scepticisme face à la méthode consistant à organiser la comparaison juridique autour de grands thèmes et en langue française. L'auteur (au demeurant parfaitement francophone) n'incrimine pas en tant que tel l'emploi de la langue française comme langue de comparaison, mais renvoie dos à dos l'usage exclusif du français et une comparaison faite "à partir du cadre et des notions de droit français " (Kitamura 1995 : 863). Autrement dit, la présentation du droit japonais - même faite en français, ou parce qu'un certain usage du français renvoie à des catégories analytiques situées - ne permettrait pas à la comparaison de se produire. Et l'auteur d'interroger la force opératoire d'une comparaison prenant le droit français comme point de départ.

Les rapporteurs japonais avaient de fait essayé d'expliquer leur propre droit " en supposant le système français comme donnée commune et en recomposant les éléments ou les arguments japonais de manière à les conformer aux notions et catégories françaises ». Cette approche consistant à " présenter ce qu'on fait au Japon, à partir et dans l'ordre du système de droit français et par les moyens français de présentation " pourrait s'expliquer par le fait des emprunts du droit japonais moderne au droit français. Ce réflexe méthodologique n'a pourtant rien d'obligé ; et l'histoire de la réception du droit au Japon n'implique pas, telle une fatalité, de repartir 
des catégories analytiques du droit français pour expliquer le droit japonais. Car, de préférence à " ce qui est bien établi, et construit ", il s'agit de " savoir ce qui est mouvant et constructeur, inquiétant et même destructeur ». Dérouler le fil de cette réflexion sur la langue de comparaison et les échanges académiques internationaux autour du droit japonais pose ainsi la question de savoir " quel est vraiment le droit japonais " (Kitamura 1995 : 865-866) - et quel est le lieu de son énonciation.

... dialogique - Soulignant l'entrée du droit comparé dans un nouvel âge, le directeur du Japan Legal Information Institute (Hō jōhō kenkyū sentā 法情報研究センター) de l'université de Nagoya insiste sur la nécessité pour le Japon, dans ses rapports avec les pays récipiendaires des programmes japonais de coopération internationale dans le domaine juridique, de se risquer d'abord à un " autoportrait» (Matsuura 2005 : 235) - moins pour ériger en modèle enjolivé le système juridique japonais, que pour le rendre (et se le rendre ${ }^{19}$ ) clairement identifiable. L'exhorte de Matsuura Yoshiharu 松浦好治 s'appuie sur sa propre expertise en matière de réforme du droit des affaires en Asie du Sud-Est : le point en question concernait les formes de sociétés reconnues en droit laotien d'une part, japonais d'autre part, donateur et récipiendaire ayant à s'expliquer mutuellement leur droit sur fond de réforme juridique croisée. Or, ce passage nécessaire par l'autoportrait est indissociable de l'exercice de traduction - sortie et projection de soi effectuée par le biais de l'anglais, "langue (étrangère) internationale ", interface entre les branches commerciales de deux droits différents. Si l'épreuve de la traduction est ici conçue comme un pas en direction de l'autre, s'en remettre à un outil linguistique commun (l'anglais, comme langue internationale) garantit-il pour autant le caractère dialogique de la comparaison ? Le cadre et les modalités de l'échange dont il est ici question - celui de la coopération internationale, souvent encore appelée "assistance juridique " ( hō seibi shien 法整備支援), et qui suppose par définition un "donateur »

19. Le coordonnateur du "Nihon hō no tōmeika 日本法の透明化 (Transparency of Japanese Law) " souligne en ce sens : "In this project, translating Japanese legislation and important judgments into English has been adopted as the principal means not only to create a database of Japanese law, but also to identify issues, which might otherwise remain unarticulated or unclear for Japanese eyes » (Kono 2009 : 229). 
et un " récipiendaire " - grèvent sans doute la réponse. La question n'en mérite pas moins d'être posée (Giraudou 2009).

\section{Le droit japonais : un droit «créole »}

Appréhender le droit japonais (moderne, voire contemporain) comme "produit de la traduction " ne revient pas à s'interroger sur le degré de conformité de la copie par rapport à son ou ses modèles supposés; mais consiste plutôt à questionner ce que la traduction y a produit. Or, aux termes de l'examen, le droit japonais n'apparaît pas seulement comme ayant été traduit, mais - et de manière plus décisive - comme étant lui-même traduction. Hasegawa Kō est le premier à avoir sondé la valeur paradigmatique de la traduction dans le droit japonais, en formulant l'hypothèse du droit japonais comme droit « créole $»^{20}$.

\section{1. Épistémologie d'un droit " créole »}

Hasegawa Kō n'est sans doute pas le seul à rappeler que la langue du droit japonais, fruit original de la traduction, est (ré)écriture. Si la modernisation du droit japonais ne consiste pas en un transplant juridique ni ne renvoie à un processus de simple réception, c'est précisément parce que l'œuvre concomitante de traduction correspond à une entreprise de (ré) écriture. Mais le philosophe du droit ne se contente pas de redire que le mode d'écrire la langue (juridique) japonaise reste affaire d'emprunt et d'adaptation. Soulevant la question de savoir dans quelle mesure la traduction renvoie à un processus de création normative, il interroge plus avant

20. Intitulé «Hō no kureōru to shutaiteki hō keisei no kenkyū 法のクレオールと主体的法 形成の研究 (A Study on the Creole and the Agency Formation of Law) », le programme de recherche dirigé par Hasegawa tente de modéliser l'intégration spatio-temporelle des échanges normatifs dans quatre " régions " (Asie du Sud-Est, Europe de l'Ouest, Amérique du Nord et Japon) : www.juris.hokudai.ac.jp/ hasegawa/lcreole/en_index. html. L'expression japonaise shutaiteki hō keisei 主体的法形成 évoque une transformation du droit qui n'a pas été subie passivement, mais qui est au contraire la manifestation d'un réel pouvoir d'initiative : une transformation initiée de manière indépendante et non pas sous la contrainte. 
cette structure qui fait "passer la pensée dans le moule des signes " : l'histoire des signes au Japon n'étant pas laissée au hasard, la langue du droit ne saurait non plus y exister « à l'aveuglette" (Herrenschmidt 2011). Bien au contraire. Faisant fond sur un système graphique distinct, dans quelle mesure la traduction (juridique) témoigne-t-elle d'une manière singulière de s'inscrire dans le monde (du droit)?

Caractériser la modernisation du droit japonais comme étant un processus de " conceptualisation isomorphique " indissociable de l'œuvre de traduction permet à Hasegawa de ne pas réduire le vocabulaire juridique à une liste baroque d'écarts traductifs, mais d'examiner différemment les multiples "sauts sémantiques" (Alcaraz Varó 2009 : 184) que favorise l'écriture japonaise et révèle le vocabulaire juridique japonais. Principale caractéristique de la traduction ainsi conçue : mise en œuvre par l'élite intellectuelle ${ }^{21}$, puis relayée par les responsables politiques, les bureaucrates et une partie de la société civile à l'époque Meiji, elle ne conduit pas à emprunter le chemin de l'imitation pure et simple. L'activité préalable de " réception personnelle " correspond plutôt à un travail d'interprétation critique $^{22}$ qui, impliquant la possibilité pour le traducteur d'être pleinement agent de l'acte d'interprétation (et non " réceptacle " d'une signification donnée a priori et non négociable), pose les bases d'un système juridique complexe, non réductible à quelque réplique de modèles étrangers.

La notion de hō shutaisei 法主体性 (en anglais : legal agency) désigne ainsi la maîtrise du processus de "réception ». Ce travail d'" incorporation sélective " permettant la constitution du système juridique japonais en une " unité organique d'idées et de valeurs hétérogènes » (Hasegawa 2009b : 89) comporte une phase de pré-interprétation qui, d'ordre analogique, consiste à effectuer un rapprochement conceptuel entre différents "schémas » et

21. Plus spécialement Mitsukuri Rinshō 箕作麟祥 (1846-1897), Nishi Amane 西周 (1829-1897), Tsuda Mamichi 津田真道 (1829-1903) et Fukuzawa Yukichi 福澤諭吉 (1835-1901).

22. Hasegawa se place du point de vue herméneutique, tel que développé par la phénoménologie et la théorie du droit comme interprétation ; il fonde son analyse en particulier sur les travaux de Hans-Georg Gadamer (Truth and Method, 1989) et de Ronald Dworkin (Law's Empire, 1986) ; Hasegawa lie également la notion d'interprétation à celle de traditions juridiques; et se réfere aux travaux de H.P. Glenn (Legal Traditions of the World, 2010). 
suppose l'existence d'un "squelette " des idées, sorte de jointure conceptuelle Est-Ouest assurant leur réceptivité dans des contextes juridiques différents. Ce processus de "reconnaissance préalable " n’implique pas une rupture avec le fond conceptuel préexistant du "pays d'accueil » : ouvert aux approximations et aux distorsions de sens, il peut aussi conduire à une certaine neutralisation de la force subversive des idées nouvelles. Ultérieure, la phase de conceptualisation consiste à conférer à l'idée étrangère une signification particulière dans la langue du pays « d'accueil », avant que celle dite d' " anticipation " n'interroge les conditions d'acceptabilité et de réalisation pratique des idées; le choix de tel vocable plutôt que tel autre indiquant à quel point cette opération s'insère étroitement dans un contexte culturel, politique et social donné.

Hasegawa écarte l'hypothèse de la sujétion - ou empire exercé par les idées étrangères - et celle, inverse, de leur rejet, mais il retient deux autres hypothèses: l'annexion et la coexistence ; selon les cas, l'opération de traduction conduit donc soit à subsumer les idées étrangères en les connectant aux idées et valeurs du pays "d'accueil ", soit à reconnaître le caractère original de ces idées par rapport aux idées et valeurs du pays en question. Là encore, les déclinaisons sont possibles, selon que l'on adopte une logique d' "inclusion " (connexion entre deux idées en vue de les ramener à un seul concept), $d^{\prime}$ " exceptionnalisation " (caractérisation des idées étrangères comme exceptions aux valeurs et idées du "pays d'accueil »), ou de "sphéricalisation " (identification et assignation différenciée des idées). La stratégie de transformation du système juridique privilégiée par les intellectuels japonais au XIX ${ }^{\mathrm{e}}$ siècle ayant été celle de l'inclusion.

\section{Les mots d'un droit " créole »}

"Empire des mots de traduction» (Kitamura 1987 : 765), le vocabulaire juridique japonais a fait l'objet de manipulations originales - qui toutes renvoient à une méthode et à un style particuliers de traduction. Ces différentes manipulations lexicales - recherches d'équivalences formelles ou d'équivalences fonctionnelles, emprunts ou transcriptions, néologismes témoignent de la complexité des mécanismes que l'approche courante, ou " croyance commune ", associe à la "réception " du droit au Japon. Or, tous les mots de traduction propres au droit japonais ne renvoient pas à un processus de "créolisation (ou recréation) normative ». Deux hypothèses 
sont ici distinguées : pour singulières soient-elles, certaines pratiques de traduction ne conduisent pas nécessairement à l'élaboration (ou à la tentative d'élaboration) d'une nouvelle langue juridique.

\section{a. Des pratiques singulières de traduction}

Un mot - plusieurs significations (et inversement) - Interrogeant l'origine du mot hō 法 (droit), Kitamura Ichirō souligne l'accumulation de sens dont le mot a fait l'objet au Japon ${ }^{23}$ et le risque consécutif de faire cohabiter dans un même vocable des concepts contradictoires. La cohérence de 法 est alors mise en doute, le syncrétisme de signifiés conduisant moins à enrichir le vocable qu'à en faire une notion-cadre au contenu indéterminé. Au-delà du mot japonais, l'éclatement concerne l'ensemble de la sphère juridique : par le biais d'une traduction épousant le cloisonnement disciplinaire, la langue japonaise du droit peut conduire à une profusion pléthorique qui fragmente l'économie interne d'une même notion. Ainsi, par exemple, les mots kabushiki 株式, soken 訴権, uttae 訴元, signifient-ils tous " action " - respectivement pour le commercialiste, le processualiste, et le civiliste. De même, kyōdō shiso 共同始祖, chosakusha 著作者, kagaisha 加害者, köisha 行為者 ou hannin 犯人 désignent l'« auteur ", respectivement en droit de la famille, en droit de la propriété littéraire, en matière de responsabilité civile, et en droit pénal. Et ainsi de suite.

Inversement, procédant non par découpage mais par contraction, il arrive qu'un même vocable serve à traduire divers synonymes. Les mots de traduction sont, dans ce cas, banalisés et homogénéisés. Par exemple, aux termes d'une traduction japonaise du Nouveau Code de procédure civile français, le

23. L'idéogramme chinois 法 (originairement 漉) exprime une double idée : peine et réglementation, d'une part ; méthode, exemple, ou standard (guidant les fonctionnaires dans l'application des ordonnances impériales), d'autre part. La traduction en Chine des sütra bouddhiques conduisit à assimiler 法 à la notion de dharma (loi suprême du cosmos et de la nature). 法 accosta au Japon avec le bouddhisme. Double de la notion chinoise du droit, la lecture japonaise du caractère a cependant conduit à assimiler 法 à nori のり/宣り (règlement " édicté " par un seigneur divin). Puis le « droit » (au sens de " droit objectif ") occidental est venu se surajouter à cette première accumulation de signifiés. Les juristes ne considèrent aujourd'hui 法 qu'au sens de "droit objectif "; les autres sens n'en restent pas moins " en fait vivants ". 
mot $\operatorname{sosh} \bar{c}$ 訴訟 désigne à la fois " procès ", « contentieux ", « recours ", « instance », « matière »; mōshitate 申立 signifie aussi bien la "prétention » que les « conclusions » ou encore le " pourvoi ». Cet effet de convergence n'est pas dommageable lorsque la variation lexicale, voulue, est principalement destinée à éviter les répétitions dans le texte source ; conduisant à niveler la différence technique existant entre différents mots proches, il devient toutefois plus gênant.

Transcriptions katakana et nouveaux kanji à lecture phonétique : refus de traduire ou tentative d'incorporation radicale? - Le traducteur japonais a parfois tenté de " traduire " des mots nouveaux en les retranscrivant à l'aide du syllabaire katakana. Par exemple, pour parer aux difficultés soulevées par la traduction du mot " liberté », certains ont proposé d'onomatopéiser la notion au lieu de l'idéogrammiser en kanji : retranscrit en katakana, リベルテ (se lit riberute) ne signifie rien en japonais courant, mais devient un " mot de passe ", voire un "guignol juridique " ou une " marionnette scientifique " (Kitamura 1987 : 776, 791) - et, en cela, d'autant plus exposé à l'effet de mystification : que le lecteur en ignore la signification n'empêche pas les mots de traduction d'avoir une valeur à ses yeux; à moins qu'ils ne lui semblent recéler une signification d'autant plus précieuse qu'elle lui est inconnue ou lui demeure hermétique.

Une approche littérale radicale fut un temps envisagée, qui devait fonctionner sur la base de kanji nouveaux à lecture phonétique. Au début de l'ère Meiji, le ministre de la Justice sollicita ainsi un érudit chinois pour créer de nouveaux idéogrammes spécialement destinés à représenter non seulement le sens mais aussi la prononciation des termes juridiques... français. Il s'agissait d'obtenir une concordance artificielle parfaite entre la terminologie française et sa "traduction " japonaise. Le projet n'a pas eu de suite, mais a laissé une trace sous la forme d'un lexique juridique répertoriant autant de " mots fantômes" (Kitamura 1987 : 766).

Combinaisons anciennes de kanji - nouvelles significations : traduction truquée ou recomposition de sens? - Il arrive au traducteur japonais de faire fond sur certains mots existants dont il va élargir ou au contraire restreindre la signification. Ainsi le mot kenpō 憲法, jusqu'alors traditionnellement employé au sens large comme désignant tout acte législatif ou réglementaire, s'est-il mis sous Meiji à signifier "Constitution ». Ainsi également de 
certains mots " connotés ", comme shihō 私法 (littéralement "droit privé »), que le shogunat qualifiait négativement comme règlementation arbitraire prise clandestinement au sein d'un groupe déterminé, et qui désigne maintenant de manière neutre une branche spécifique du droit. Ainsi encore du mot jiyz 自由, qui ne constitue pas à proprement parler un néologisme : traditionnellement associé à l'arbitraire et à l'égoïsme, il sera toutefois repris par le traducteur sous Meiji pour traduire « liberté ». Et ainsi de suite.

Dans chacun de ces cas, on constate un effet de valorisation : traditionnellement connoté de manière négative, le mot existant - devenu support de traduction - se voit doté d'une valeur nouvelle. Cette manipulation comporte un risque : pour le lecteur, le mot continuera peut-être de véhiculer aussi son sens lié à la tradition. Or, les répercussions d'un tel « effet de valorisation " sont loin d'être anecdotiques. S'agissant du mot de traduction jiyz, une compréhension à partir de la notion japonaise traditionnelle risque de conduire à une dévalorisation de la "liberté ", tandis qu'une compréhension à partir de la " liberté " peut conduire à survaloriser jiyzu. D'où mise en garde contre ce " trucage inconscient " consistant pour les intellectuels à « lire artificiellement la présence de la "liberté" dans le contexte japonais, sans voir effectivement les aspects réels de "jiy $\bar{u}$ " " (Kitamura 1987 : 775).

\section{b. Une tentative de recréation normative}

Les tentatives de recréation normative, ou d'élaboration d'une nouvelle langue juridique, sont au Japon intimement liées à des mécanismes traductifs distincts des techniques ci-dessus envisagées. Exemplaire à cet égard est le processus qui conduira les intellectuels de Meiji à traduire rights par kenri 権利 - en référence à l'idée de pouvoir, comme prôné par Nishi Amane 西周 (1829-1897) et Tsuda Mamichi 津田真道 (1829-1903) - de préférence à tsūgi 通義, initialement suggéré par le réformateur Fukuzawa Yukichi 福澤 諭吉 (1835-1901), en référence à l'idée de justice, voire de juste raison. Cette élite intellectuelle dont les traducteurs font partie dans le Japon de la fin de l'époque d'Edo et du début de l'ère Meiji, approchera les droits par leur versant le plus aisément identifiable à l'époque : la traduction du néerlandais regt comme de l'anglais right puise à une certaine compréhension des relations de pouvoir au sein de la société et reste indissociable de la recherche d'un aménagement équilibré des relations d'autorité. La 
place manquant ici, ne sont ici abordés que les principaux aspects, renvoi étant fait à mon étude préliminaire pour une présentation plus détaillée (Giraudou 2011a).

Dans le premier des textes s'attachant à une traduction du néerlandais regt (Tonarigusa 隣草, 1861), Katō Hiroyuki 加藤弘之 (1836-1916) emploie ken 権, ou "pouvoir de l'État ". Un deuxième usage de ken renvoie à la division tripartite des pouvoirs au sein de l'État, plusieurs auteurs sous Meiji utilisant ken pour désigner les trois grands pouvoirs (législatif, exécutif, judiciaire), comme dans l'expression san dai ken 三大権. Dans les écrits de Nishi Amane et de Tsuda Mamichi, ken désigne le " pouvoir souverain » ou la «souveraineté » (shuken 主権). Outre les droits de l'État, ken désigne les droits (en termes de pouvoir et d'autorité) de ses fonctionnaires : ainsi notamment des mots kenpei 権柄 (pouvoir de contrôle), kensei 権 勢 (pouvoir, autorité, empire), ken.i 権威 (autorité, prestige, compétence), tokken 特権 (pouvoirs spéciaux ou privilèges). Introduite et reproduite au Japon à partir de 1865 sous le titre Bankoku kōhō 万国公法, la traduction en chinois de l'ouvrage Elements of International Law with a Sketch of the History of the Science, écrit en 1836 par l'américain Henry Wheaton, utilise aussi le caractère 権 combiné avec d'autres caractères chinois pour traduire notamment les "privilèges » attachés à la charge d'ambassadeur. Au cours des années 1870 , la plupart des auteurs se mirent à utiliser les mots kenri 権利 (droits) et gimu 義務 (devoirs) - d'usage courant encore aujourd'hui. Ont leur part dans la diffusion large de ces deux vocables les traductions très médiatisées de On Liberty (J. S. Mill) par Nakamura Masanao 中村 正直 dit Keiu 敬宇 (1832-1891) en 1871, et d'Allgemeines Staatsrecht (J. K. Bluntschli) par Katō Hiroyuki en 1872, ainsi que les tentatives de ce dernier de réaliser une synthèse entre le constitutionnalisme occidental et les traditions japonaises.

Fukuzawa Yukichi proposait cependant une traduction moins centrée sur l'idée de pouvoir. En 1870, il suggère ainsi de traduire rights et duty respectivement par les néologismes tsügi 通義 (justice, commune juste raison) et shokubun 職分 (charge, devoir). Le choix de tsūgi pour traduire rights souligne le caractère juste du titre à réclamer et est lié à cette compréhension de l'autonomie individuelle et de l'égalité de tous devant la loi qui le conduira à identifier plus finement que d'autres intellectuels et traducteurs de l'époque le rapport entre droits et forme républicaine 
de gouvernement ${ }^{24}$. Certes, Fukuzawa aussi utilise ken 権 dans ses formes composées. En 1873, il propose ainsi de traduire rights par kengi 権義, une combinaison de caractères désignant " pouvoir " (ken 権) et " juste raison" ( $g i$ 義), puis par kenri 権理 (désignant respectivement " pouvoir » et " raison »). Ces expressions s'accordent toutes sur un point : la réclamation est fondée, et s'accompagne d'un pouvoir, ou autorité, pour autant qu'elle présente un caractère de rectitude ou de raisonnabilité.

Dans les années 1880, kenri 権理 et kenri 権利 se le disputaient encore, avant que ne finisse par l'emporter le choix de kenri 権利 (combinaison de caractères signifiant respectivement " pouvoir " et "intérêt ») pour traduire rights. Kitamura Ichirō observe que "le droit subjectif est ainsi réduit à l'intérêt que les particuliers peuvent tirer du droit, du moins littéralement parlant "; la séparation entre droit objectif (ramené aux commandements de l'État et à son appareil de contrainte) et droit subjectif (assimilé à la puissance et à l'intérêt) conduisant en outre à « ne jamais les retrouver dans une même configuration primordiale de ce qui est juste " (Kitamura 1987 : 777, 787). Noda Yoshiyuki souligne de même que « à l'heure actuelle, presque tout le monde ignore que ce mot [kenri 権利, ou "droit" au sens de droit subjectif] implique le sens d'impartialité "; en effet, "pour le Japonais moyen, ce mot évoque quelque chose d'égoïste ». Et d'interroger : les Japonais connaissent-ils « vraiment ce que le Français entend par le droit ? » (Noda 1966 : 175). Selon Hasegawa, l'idée exprimée par la combinaison des caractères tsügi 通義 était sans doute trop radicale pour l'imagination autoritariste des Japonais de l'époque - et, de fait, la préférence ira très largement à une traduction plus en phase avec la manière, au fond traditionnelle, dont est conçu le réaménagement des rapports d'autorité au sein de la société (harmonisation des réclamations du fort et du faible, indépendamment de la rectitude de leurs demandes respectives) (Hasegawa 2009b). Mais qu'ils soient formulés sur le mode radical, sur celui du compromis, ou de manière plus immédiatement consensuelle, les mots tsügi 通

24. Or, dans le contexte d'un régime autoritaire, la conception positiviste des droits comme pouvoirs juridiquement reconnus devait conduire à appréhender ces derniers en termes de droits octroyés par une autorité supérieure, ou de " commandements juridiques » - et donc à éclipser davantage encore l'idée d'un titre légitime à formuler une réclamation juste. 
義, kenri 権理 ou kenri 権利 n'en participent pas moins d'un processus complexe d'interprétation, d'aménagement-reconstruction, voire de création, indissociable de l'opération de traduction.

\section{Le droit " créole " : un espace d'articulation normative ?}

En restituant à ce jeu d'influences multiples son caractère complexe, l'analyse du droit japonais comme traduction - creuset d'une " créolisation normative " - rendrait-elle définitivement caduques certaines présentations comparées classiques ? Ce faisant, participerait-elle de cette tentative de penser (en droit japonais, et le droit japonais comme) la réconciliation de différentes traditions, pratiques et pensées juridiques? Et en quoi permettraitelle d'ouvrir la réflexion juridique - non plus seulement comparée, mais aussi internationale - à la notion d'articulation normative?

\section{a. Renouvellement de la réflexion sur la "réception juridique »}

Soucieux d'appréhender plus finement la complexité des échanges normatifs et la singularité de l'expérience japonaise en matière de réception du droit, un petit nombre de juristes japonais dénoncent l'insuffisance des taxinomies classiques du droit comparé et s'attachent à repenser les méthodes mêmes du comparatisme. Non sans contradictions, certains auteurs tentent ainsi de renouveler les termes de la réflexion sur la réception du droit au Japon en adoptant la perspective des systèmes juridiques dits " mixtes ${ }^{25}$ ". Dans quelle mesure l'approche du droit japonais comme droit " créole " s'articule-t-elle à cette tentative de repenser le comparatisme juridique au Japon $^{26}$ ?

Le paradigme du droit comme traduction ne se ramène pas à l'hypothèse de la traductibilité. Là réside son principal intérêt. La question du métraduit (irruption de l'inattendu, voire du malentendu) et de l'intraduit (refus ou

25. Ainsi que l'a expliqué Matsumoto Emi dans son intervention orale ("Japanese Law as a Mixed Legal System ") au colloque international Global Law and Global Legal Theory-Academic Knowledge in Question, op. cit. note 3.

26. Telle était l'une des questions de fond soulevées lors du colloque international précité. 
impossibilité de traduire, absence délibérée ou subie de traduction) pourrait, certes, se poser (Legrand 2005). Mais un tel questionnement - notamment en ce qu'il continue d'appréhender la traduction sur le mode dichotomique (d'une langue source radicalement étrangère à une langue cible strictement autochtone) - ne fait que balancer entre deux extrêmes : impossibilité même pour le droit de se traduire et donc de « circuler " d'un système à un autre ; ou circulation à partir d'un centre (invariablement ancré à l'ouest), via transplants. Placer le processus de "créolisation " au cœur même du droit japonais permet au contraire de dépasser le questionnement binaire traduisible/intraduisible, traduit/non traduit, et de poser en d'autres termes la question du droit et de la création normative. Car, comme le souligne Hasegawa, la logique qui sous Meiji préside au travail d'interprétation critique, reste bien une logique d'annexion, non pas de coexistence. Son analyse éclaire, en définitive, combien la continuité entre différents systèmes et traditions juridiques permettant la transmission d'idées ou de concepts, n'est pas directe. En s'attachant à décrire «la production d'équivalence (sans adéquation) par la traduction ", lui aussi appelle à " comparer l'incomparable ", précisément en construisant des comparables. Ce qui suppose de dépasser l'approche strictement positiviste et d'élargir le cadre théorique à un ensemble de disciplines juridiques : au-delà des approches statiques de droit comparé développées jusqu’à présent ; au-delà des études en histoire du droit, trop souvent limitées à une analyse exégétique des sources ; au-delà des approches anthropologiques du droit, centrées sur l'opposition droit moderne/droits pré-modernes.

Faire fond sur la "créolité " pour appréhender le droit, ses transformations, et la science juridique elle-même, donne ainsi de rompre avec la conception du système japonais comme " œuvre d'une greffe par traduction " (Kitamura 1987 : 791). En brisant les schémas de pensée inspirés des sciences de la nature, le paradigme de la traduction permet en effet de dépasser l'hypothèse classique d'une modernisation juridique effectuée via de simples transplants juridiques (d'Ouest en Est). Si l'approche du droit comme traduction interroge la notion de "réception ", c'est bien plutôt à partir de ces moments particuliers que sont ceux de création normative ou d'innovation juridique. Consécutivement, le concept de "créolité " permet de quitter le terrain confortable d'une réception juridique principalement conçue comme superposition d'influences successives : de nature à éclairer la complexité spatio-temporelle des processus de transformation du droit, 
le cadre théorique élaboré par Hasegawa permet de prendre l'exacte mesure de ces mécanismes de (re)création normative qui au Japon sont intimement liés à des processus traductifs et, ce faisant, de caractériser en quoi le droit japonais est un "droit hybride" (Hasegawa 2003).

\section{b. Ouverture de la réflexion à la notion d' "espace normatif "}

En un temps de " porosité juridique " renforcée, dans quelle mesure la notion de « créole » en droit permettrait-elle de rendre compte de la transformation continue des droits et du degré de complexité avérée des échanges juridiques ? L'analyse des mots du droit a pu inciter certains comparatistes à reconstituer des systèmes, voire des familles, en établissant des liens de filiation - toujours dans un but de reconstruction du réel juridique (et le plus souvent à des fins idéologiques) (Richard 2007 : 92). L'incursion du paradigme de la traduction dans le droit revêt pourtant une tout autre portée, et vient enrichir l'approche critique des classifications traditionnelles du droit comparé. Concernant le droit japonais, il ne s'agit pas tant de mesurer - à partir de critères linguistiques et autres recoupements lexicaux - l'influence de tel droit (système ou famille juridiques), que de replacer la modernisation de ce droit et sa réforme actuelle dans un contexte de globalisation au croisement même d'un ensemble de pratiques, de normes et d'idées juridiques.

C'est pourquoi l'hypothèse selon laquelle l'ouverture géographique et conceptuelle des espaces de régulation à l'époque contemporaine serait indissociable de l'émergence d' " espaces normatifs » conçus comme autant d' "espaces langagiers communs", ouvre pour ma recherche des perspectives intéressantes. Telle est l'hypothèse formulée dans le cadre du programme de recherche intégré " Globex - Recherches pluridisciplinaires sur la mondialisation du droit : contrats globaux et nouvelles régulations sociales ${ }^{27}$ ", initié et coordonné par Gilles Lhuilier. Ce programme, auquel je participe, tente de vérifier l'hypothèse d'une "langue internationale des contrats " propre à de nouveaux espaces normatifs : non réductibles aux seuls "systèmes " ou " ordres " juridiques, émancipés des traditionnelles " aires juridiques

27. En ligne : http://www.mshb.fr/accueil/la_recherche/pole_gouvernance_dans_les_ institutions_publiques_et_privees/globex. 
continentales " (Common Law, Europe, Afrique, Asie, ...) sur lesquelles se concentrent les approches statiques de droit comparé, ces " espaces » intègrent partiellement ou "syncrétisent " différents éléments de droit positif, mécanismes, pratiques, pensées juridiques, et niveaux normatifs. Destiné à préciser la notion d' " espace normatif ", ce programme intéresse directement ma recherche dans la mesure où il permet d'explorer plus avant la portée du paradigme de droit comme traduction.

En lien avec les travaux développés par certaines équipes de recherche japonaises et mes activités d'enseignement, ma recherche explore en outre quelques premières pistes pour une articulation - pratique - des mots de traduction sous la forme d'un glossaire juridique multilingue dans un domaine spécifique. Un tel outil peut permettre de mieux identifier puis de connecter - d'une langue à une autre - ces « équivalences sans adéquation " qui sont autant d'innovations juridiques, de créations normatives, d'" espaces singuliers" (de régulation et de signification) au sein de différents systèmes juridiques ${ }^{28}$. La question se pose plus spécifiquement des modalités de la traduction : du japonais au français ("voie directe »), ou $\mathrm{du}$ japonais en repassant par l'anglais ("voie triangulaire "), langue internationale prioritairement choisie par les programmes officiels de traduction récents. La voie traductive directe est tentante à plus d'un titre. Opter pour cette voie n'en consiste pas moins à interroger d'abord un possible lignage entre deux systèmes juridiques, au risque d'appréhender la circulation normative de manière rectiligne. Plus acrobatique, la "voie traductive triangulaire » rendrait-elle mieux compte de la complexité des processus de création normative?

Les membres du programme Nihon hōrei eiyaku purojekuto 日本法令英訳 プロジェクト eux-mêmes suggèrent de coordonner, à terme, leur travail de traduction en langue anglaise avec les initiatives prises dans d'autres pays pour traduire de l'allemand, du français, du chinois, ou du coréen en anglais - l'idée étant de parvenir à une traduction anglaise harmonisée de ces « idées similaires des systèmes de droit civil " (Kashiwagi 2007 : 226). Cette configuration lexicale semble adopter une conception plane de la

28. Un ouvrage en cours d'écriture avec Gilles Lhuilier prévoit ainsi d'annexer une table d'équivalences lexicales multilingue ainsi qu'un glossaire japonais-français du droit japonais des sociétés. 
traduction juridique (et du droit), en ramenant tout uniment un ensemble de systèmes à la " tradition civiliste »; et paraît confirmer la centralité de la langue anglaise, étalon de mesure qui plus est érigé en " passeur » : langue cible devenue convergente, l'anglais permettrait de connecter entre eux le français, l'allemand, le chinois, le coréen et le japonais. Ce en quoi une telle configuration lexicale pourrait tourner court... Ce projet traductif multilingue n'a cependant pas pour objet de parvenir à une langue unique, sorte de point focal, mais vise une traduction commune faisant fond sur différentes approches " nationales » de notions juridiques sinon " similaires », du moins apparentées, en tout cas... rendues comparables. Ce travail implique un déplacement : le traducteur ne regarde plus en direction de la seule langue cible, en l'occurrence l'anglais, mais se trouve conduit à multiplier au préalable les angles de vue - la traduction juridique du japonais en langue anglaise s'effectuant alors de manière médiate, au prisme d'autres langues juridiques. Le dialogue traductif s'effectue ici en amont, moyennant rediscussion coordonnée entre traducteurs et, éventuellement, reformulation plus ou moins partielle. Les traducteurs ne sont certes pas au bout de leur peine, mais la difficulté prévisible d'une telle opération n'annule pas la pertinence du projet; et la proposition, qui en soi ne manque pas d'audace, retient d'autant plus notre attention qu'elle est précisément le fait de juristes japonais. 


\section{Bibliographie}

ALCARAZ VARÓ Enrique, 2009

"Isomorphism and Anisomorphism

in the Translation of Legal Texts ",

in F. OLSEN, A. LORZ, D. STEIN (eds.),

Translation Issues in Language and Law,

London, Palgrave Macmillan,

pp. 182-192.

BERMAN Antoine, 1984

L'épreuve de l'étranger, Paris, Gallimard.

\section{BROTHERSTON Gordon, 1998}

"Script in Translation », in Mona

BAKER (ed.), Routledge Encyclopedia

of Translation Studies, London,

Routledge, pp. 211-218.

BRAND Oliver, 2009

« Language as a Barrier to Comparative Law », in F. OLSEN, A. LORZ,

D. STEIN (eds.), Translation Issues

in Language and Law, London, Palgrave

Macmillan, pp. 18-34.

CAPITANT Henri (dir.), 1930

Vocabulaire juridique, Paris, PUF.

CORNU Gérard, 2000

Linguistique juridique, Paris,

Montchrestien.

\section{CORNU Marie}

\& MOREAU Michel (dir.), 2011

Traduction du droit et droit de la traduction, Paris, Dalloz.

DÉTIENNE Marcel, 2000

Comparer l'incomparable, Paris, Éd. du Seuil.
DWORKIN Ronald, 1986

Law's Empire, Cambridge, MA, Harvard University Press.

FERRIER Michaël, 2009

La barrière des rencontres, Nantes, Éditions Cécile Défaut.

GIRAUDOU Isabelle, 2009

«Les programmes japonais

de coopération internationale dans le domaine juridique : une contribution à l'approche pluraliste de la circulation du droit ? », Revue Transcontinentales, 7 : 47-67.

GIRAUDOU Isabelle, 2011a

« Le droit japonais comme traduction. Une rupture épistémologique dans le comparatisme juridique », Les Cahiers d'Ebisu (Occasional Papers), 2 : 9-72.

GIRAUDOU Isabelle, 2011b « In Search of a Formula for Legal Comparative Analysis ", Report on the International Workshop « Global Law and Global Legal Theory - Academic Knowledge in Question » (MFJ, Tokyo, 3-4 juin 2011), pp. 30-35. Document en ligne : http ://www.mfj.gr.jp/web/ sympo_20110603/pdf/CR-IW_3-4_ June_2011.pdf.

\section{GLENN Patrick H., 2010}

Legal Traditions of the World, Oxford, Oxford University Press.

De GROOT Gérard-René, 1987

«The Point of View of a Comparative 
Lawyer », Les Cahiers de Droit, 28 (4), La traduction juridique : 793-812.

GROSSFELD Bernhard, 2003

"Comparatists and Languages », in P. LEGRAND \& R. MUNDAY, Comparative Legal Studies : Traditions and Transitions, Cambridge, Cambridge University Press, pp. 154-194.

GROSSFELD Bernhard, 2005

Core Questions of Comparative Law,

Durham, Carolina Academic Press.

\section{GADAMER Han-Georg, 1989}

Truth and Method, New York, Crossroads.

\section{KISHEL Uwe, 2009}

«Legal Cultures - Legal Languages », in F. OLSEN, A. LORZ, D. STEIN (eds.), Translation Issues in Language and Law, London, Palgrave Macmillan, pp. 7-17.

\section{LANGER Maximo, 2004}

« From Legal Transplants to Legal Translations » (en particulier : point V), Harvard International Law Journal, $451: 1-65$.

\section{HASEGAWA Kō, 2003}

"The Structuration of Law and Its Working in the Japanese Legal System ", in J. VANDERLINDEN et al. (eds.), La structure des systèmes juridiques, Bruxelles, Bruylant, pp. 325-330.

\section{HASEGAWA Kō 長谷川晃, 2007}

"Hō no kureōru no gainen o meguru kisoteki kōsatsu » 法のクレオールの概 念をめぐる基礎的考察 (Considérations sur le concept de droit « créole»), The
Hokkaido Law Review (Hokudai hōgaku ronshū 北大法学論集), 58 (3) : 1310-1335.

HASEGAWA Kō, 2009a

« Incorporating Foreign Legal Ideas through Translation », in A. HALPIN \& V. ROEBEN, Theorising the Global Legal Order, Oxford, Hart Publishing.

\section{HASEGAWA Kō, 2009b}

"Between Rights and Kenri », in E. CASHIN-RETAINE (ed.), Legal

Engineering and Comparative Law, vol. 2, Schulthess, pp. 87-103.

HERRENSCHMIDT Clarisse, 2009-2010 «À propos de l'ouvrage Les trois écritures: langue, nombre, code", in Rada IVEKOIC (dir.), Revue Asylon(s), 7, "Que veut dire traduire? ». En ligne sur TERRA, réseau scientifique de recherche et de publication.

\section{HERRENSCHMIDT Clarisse, 2007}

Les Trois Écritures : langue, nombre, code, Paris, Gallimard.

KAMEI Takashi 亀井孝, ŌTŌ Tokihiko 大藤時彦, YAMADA Toshio 山田俊雄 (eds.), 1963 Nihongo no rekishi 2 : Moji to no meguriai 日本語の歴史 (2) 文字とのめぐりあい

(Histoire de la langue japonaise, t. 2 : La découverte des caractères), Tokyo, Heibonsha 平凡社.

KASAI Yasunori 葛西康徳, 2009

«Koko ga hen da yo Nihon hō ここが 変だよ日本法 Is Japanese Law a Strange Law? ", Reports and Summarized Contributions of the Symposium (Tokyo 
28-29 nov. 2008), ZJapanR (Journal for Japanese Law), 28 : 6-13.

KASAI Yasunori 葛西康徳, 2010

« Hō no tōmeika purojekuto e no hikakuhō — Hōseishi kara no o kaeshi » 法の透明化プロジェクトへの比較法 一一法制史からのお返し (Le droit comparé au regard du « Transparency of Japanese Law Project » : la dette de l'histoire du droit), Juristo ジュリスト, 1394 : 29-36.

\section{KASHIWAGI Noboru, 2007}

"Translation of Japanese Statutes into English », ZJapan/RJ.Japan.L, $23: 221-226$

\section{KITAMURA Ichirō, 1987}

"Un point de vue japonais », Les Cahiers de Droit, 28 (4), La traduction juridique : 747-792.

KITAMURA Ichirō, 1995

«Brèves réflexions sur la méthode de comparaison franco-japonaise", Revue internationale de droit comparé, 47 (4) oct.-déc. : 861-869.

\section{KOTHARI Rita}

\section{\& WAKABAYASHI Judy, 2009}

"Introduction ", in Judy WAKABAYASHI \& Rita KOTHARI (eds.), Decentering Translation Studies, Amsterdam, John Benjamins Publishing Company, pp. 1-16.

\section{LEGRAND Pierre, 2005}

"Issues in the Translatability of Law ", in S. BERMAN \& M. WOOD (eds), Nation, Language and the Ethics of Translation, Princeton, Princeton

University Press, pp. 30-50.

LEVY Indra (ed.), 2011

Translation in Modern Japan, London, New York, Routledge.

\section{LHUILIER Gilles, 2008}

La loi, roman, Québec,

Presses universitaires de Laval.

MARUYAMA Masao 丸山真男

\& KATŌ Shūichi 加藤周一 (eds.), 1991

Hon.yaku no shisō 翻訳の思想

(Les idéologies de la traduction),

Tokyo, Iwanami shoten 岩波書店.

MARUYAMA Masao 丸山真男 \& KATŌ Shūichi 加藤周一 (eds.), 1998 Hon.yaku to Nihon no kindai 翻訳と 日本の近代 (Traduction et modernité au Japon), Tokyo, Iwanami shoten 岩波書店.

MATSUMOTO Emi, 2010

" Lost in Translation : the Reception of German Law in Japan », Hōsei Riron 法政理論 (The Journal of Law and Politics - Niigata University), 43 (3-4) : 110-128.

MATSUURA Yoshiharu, 2005

« Improving Legal Transparency:

The Translation Project of Japanese Law and the Prospect of New Comparative Law », in MATSUURA Y. (dir.) The Role of Law in Development: Past, Present and Future, Nagoya, Nagoya University.

MIZUTA Norihisa 水田紀久,1968 «Nihon kangaku no tenkai »日本漢学の 展開 (Le développement de la sinologie au Japon), in MIZUTA N. (ed.), Chūgoku 
bunka sōsho (Dai 9 kan) Nihon kangaku 中国文化叢書 (第9巻) 日本漢学， Tokyo, Taishūkan shoten 大修館書店， pp. 272-284.

\section{NELKEN David, 2002}

"Legal Transplants and Beyond: of Disciplines and Metaphors", in A. HARDING A. \& E. ÖRÜCÜ (dir.), Comparative Law in the 21st Century, The Hague, Kluwer, pp. 19-34.

\section{NELKEN David, 2003}

"Comparatists and Transferability », in P. LEGRAND \& R. MUNDAY, Comparative Legal Studies: Traditions and Transitions, Cambridge, Cambridge University Press, pp. 437-466.

\section{NODA Yoshiyuki, 1966}

Introduction au droit japonais, Paris, Dalloz.

NODA Yoshiyuki 野田良之, 1975

Gendai shōhō gaku no kadai 現代商法学の課題(中) (Études offertes à Takeo Suzuki - Problèmes de droit commercial moderne), t. 2, Tokyo, Yūhikaku 有斐閣, pp. 689-717.

\section{NODA Yoshiyuki 野田良之, 1979}

«Kenri to iu kotoba ni tsuite » 権利という言葉について (À propos du mot « droits »), Gakushūin daigaku hōgakubu kenkyū nenpō 学習院大学法 学部研究年報 (Annales de la Faculté de droit de l'université Gakushūin), $14: 1-30$.

\section{ÖRÜCÜ Esin, 2002}

"Unde Venit, Quo Tendit Comparative
Law? », in A. HARDING A. \& E. ÖRÜCÜ

(dir.), Comparative Law in the 21st

Century, The Hague, Kluwer : 1-17.

POUND Roscoe, 1954

«Some Thoughts about Comparative Law », in H. DÖLLE (ed.), Festschrift for Ernst Rabel, Tübingen, Mohr, pp. 7-16.

\section{RICHARD Pascal, 2007}

Le jeu de la différence. Réflexions sur l'épistémologie du droit comparé, Québec, Les Presses universitaires de Laval.

RICCEUR Paul, 2004

Sur la traduction, Paris, Bayard.

SACCO Rodolpho, 2011

"Aperçus historique et philosophique des relations entre droit et traduction ", in Marie CORNU \& Michel MOREAU (eds.), Traduction du droit et droit de la traduction, Paris, Dalloz.

\section{ŠARČEVIĆ Susan, 1998}

"Creativity in Legal Translation:

How Much is too Much? », in A. CHESTERMAN, N. G. SAN SALVADOR, Y. GAMBIER (eds.), Translation in Context, Amsterdam, John Benjamins Publishing Company, pp. 281-292.

\section{SEMIZU Yukino, 2006}

«Invisible Translation: Reading Chinese Texts in Ancient Japan », in Th. HERMANS (ed.), Translating Others, Manchester, St. Jerome Publishing, pp. 283-295. 
STEINER George, 1975 (1992)

After Babel: Aspects of Language and

Translation, Oxford, Oxford University

Press.

SUGITA Genpaku 杉田玄白, 1815 (1982)

Rangaku koto hajime 蘭学事始 (Mémoire sur les premières années de nos études hollandaises), Tokyo, Iwanami shoten 岩 波書店.

TAKII Kazuhiro 瀧井一博, 2003

Bunmeishi no naka no meiji kenpō. Kono kuni no katachi to seiyō taiken 文明史の 中の明治憲法一この国の形と西洋体験， Tokyo, Kōdansha 講談社, 2003 ; traduction anglaise de David NOBLE 2007 : The Meiji Constitution, The Japanese Experience of the West and the Shaping of the Modern State, Tokyo, International House of Japan.

TEPLOVA Natalia (dir.), 2009 « La traduction au Japon/Translation in Japan », TTR. Traduction, Terminologie, Rédaction, 22 (1).

UEDA Atsuko, 2011

"Sound, Scripts, and Styles: Kanbun kundokutai and the national language reforms of 1880s Japan », in Indra LEVY (ed), Translation in Modern Japan, London, New York, Routledge, pp. 133-156.

\section{UEHARA Mayuko, 2009}

«Vers une convergence de la traduction et de la philosophie dans le Japon moderne », in T. Milliaressi (dir.), La traduction: philosophie, linguistique et didactique, Lille, Éditions du Conseil scientifique Université Charles de

Gaulle : 55-58.

UEHARA Mayuko, 2010

«The Philosophy of Translation:

From Nishida Kitarō to Ogyū Sorai », in J.W. HEISIG \& R. RAUD (eds.), Frontiers of Japanese Philosophy 7. Classical Japanese Philosophy, Nanzan Institue for Religion \& Culture, pp. 305-319.

WAKABAYASHI Judy, 2005

"The Reconceptualization of Translation from Chinese in Eighteenth-century Japan », in Eva HUNG (ed.), Translation and Cultural Change, Amsterdam, John Benjamins Publishing Company, pp. 121-145.

YANABU Akira, 2009

«Translation in Japan : The Cassette Effect », TTR. Traduction, Terminologie, Rédaction, 22 (1) : 19-28.

YANABU Akira 柳父章, 1982 Hon.yakugo seiritsu jijo 翻訳語成立事情 (Les circonstances de la formation des mots de traduction), Tokyo, Iwanami shinsho 岩波新書. 